\title{
Hypothermia, CTCAE
}

National Cancer Institute

\section{Source}

National Cancer Institute. Hypothermia, CT CAE. NCI Thesaurus. Code C55332.

A disorder characterized by an abnormally low body temperature. Treatment is required when the body temperature is $35 \mathrm{C}$ (95F) or below. 